\title{
Foamy myocardial transformation of infancy: an inherited disease
}

\author{
V SUAREZ,* W J FUGGLE, $†$ A H CAMERON, $\dagger$ T A FRENCH,* \\ T HOLLINGWORTH $\ddagger$
}

From the Department of *Histopathology, Central Pathology Laboratory, Stoke-on-Trent, the Department of $\dagger$ †istopathology, Birmingham Children's Hospital, and the $\ddagger$ Cellular Pathology Department, Russells Hall Hospital, Dudley, Birmingham

SUMMARY Five cases of foamy myocardial transformation of infancy, a condition which predominantly affects female children under 2 years of age and which causes cardiac arrhythmia or sudden death, are reported. Of these five cases, four occurred in two sets of siblings, suggesting a possible hereditary basis for the disease. As far as we know, no other familial cases have been reported. The other case was of focal disease of the myocardium, as opposed to the diffuse myocardial changes seen in the four familial cases.

Voth $^{1}$ first recognised this rare condition in 1962 and defined it as arachnocytosis of the heart muscle. In 1965 Hudson $^{2}$ referred to a child who had died in atrial tachycardia and whose heart showed an unusual form of lipid storage disease with no other organ affected.

The cases reported have been predominantly in females and under 24 months of age. Various arrhythmias, mainly tachycardias, have been detected before death in several cases. Sudden death in an apparently healthy infant, however, has been documented in several others.

At necropsy the heart is enlarged. Histological examination shows myocytes with a swollen, foamy, and granular cytoplasm. Ultrastructural studies have shown that this granularity is due to increased numbers of enlarged mitochondria.

The aetiology is unknown, but a genetic basis has been proposed, based on the strong female preponderance. ${ }^{3}$ The following case reports add further evidence to a hypothesis of a genetic aetiology.

\section{Case reports}

\section{CASES 1 AND 2}

Two children were born to caucasian nonconsanguineous parents (family A), were separated by an eight year interval. A third sibling, a 7 year old male, at the time of death of the second child, is alive and well at the time of writing.
Case 1

A 4 month old female infant was admitted to hospital with a history of failure to thrive and in severe heart failure. She was pale but not cyanosed, with a systolic murmur at the left sternal border. The pulse rate was $160 /$ minute. She died eight days after admission in severe congestive cardiac failure.

Case 2

An 8 month old baby girl, sibling of case 1, was admitted to hospital in congestive cardiac failure. She had been completely well until six days previously, when she became sweaty and had diarrhoea. An electrocardiogram performed on admission showed a supraventricular tachycardia with a rate of $155 /$ minute. She died 24 hours later.

\section{CASES 3 AND 4}

Cases 3 and 4 were two of five children born to caucasian non-consanguineous parents (family B). There are no surviving children from this marriage. Two pregnancies resulted in spontaneous abortions at 12 and 20 weeks' gestation. Another sibling died of encephalitis aged six months and this child had no evidence of cardiac disease at necropsy.

Four children from previous marriages and liaisons are all alive and well at the time of writing.

\section{Case 3}

Case 3 was a 16 month old male infant who had previously been well and suddenly collapsed and died at home. 


\section{Case 4}

Case was a 4 month old male infant who had been unwell with a cough and loss of appetite for five days prior to admission. He had a severe pallor but no fever. His pulse rate was $140 /$ minute, and there were no cardiac murmurs. There was moderate subcostal recession, but his breath sounds were vesicular with no added noises. Respiratory infection with possible septicaemia was diagnosed. He died 10 hours later after a cardiac arrest.

\section{CASE 5}

An 18 month male infant born to caucasian nonconsanguineous parents had been unwell for a few days with a rash and one episode of vomiting. His general practitioner found that the child was sweaty and feverish with a rash over the trunk. The chest was clear. Measles was diagnosed. Two days later he was found dead in his cot by his mother. Three other siblings now aged 10,3 , and 2 years are all well at the time of writing.

\section{Methods}

Random blocks from the free wall of the left ventricle (cases 1-5), right ventricle and interventricular septum (cases 1-4), and both atria (cases 1, 2, and 4) were either fixed in formol saline (cases 1 and 2) or buffered formalin (cases 3, 4, and 5). Specific blocks to show the conducting system were not taken. Best's Carmine stain (case 2) was used on frozen section and alcohol fixed sections. Two mitochondrial stains were used in case 2 . The first was on formalin fixed tissue postfixed in dichromate and stained by Altmann's acid fuchsin-picric acid technique and the second was a block staining method.

In the latter method blocks of formalin fixed washed tissue were placed in $1 \%$ osmium tetroxide in the dark for seven to 14 days, with two changes of osmium during that time. After washing in distilled and tap water the blocks were dehydrated, cleared, and embedded in paraffin wax - cut and mounted in the usual way. Mitochondria were shown as black granules. This gave the clearest light microscopic impression of the numbers of mitochondria.

Tissue from the free wall of the left ventricle for electron microscopy (case 2) was fixed in $2.5 \%$ phosphate buffered gluteraldehyde plus $5 \%$ sucrose, postfixed in $1 \%$ osmium tetroxide, processed and embedded in TAAB resin, cut and stained in uranyl acetate and lead citrate; and viewed with an AE1 801 microscope. In case 4 tissue from the free wall of the left ventricle for electron microscopy was fixed in phosphate buffered formalin, post-fixed in gluteraldehyde, and secondarily fixed in osmium tetroxide. Tissue from the paraffin blocks in case 3 was
Table Heart weights at necropsy

\begin{tabular}{lrll}
\hline Case no & Age (months) & Heart weight $(\mathrm{g})$ & Normal for age $(\mathrm{g})^{4}$ \\
\hline 1 & 4 & 100 & 27 \\
2 & 8 & 114 & 37 \\
3 & 16 & 120 & 48 \\
4 & 4 & 88 & 27 \\
\hline
\end{tabular}

dewaxed and secondarily fixed in osmium tetroxide. Tissue of cases 3 and 4 was then processed and em- $\omega$ bedded in Emix resin, stained with uranyl acetate and $\overrightarrow{0}$ lead citrate, and viewed with an AEl Corinth $500 \mathrm{mi}-$ croscope. Tissue was excised from the paraffin block in case 5, dewaxed, and treated as case 2 . Electron microscopy was not performed in case 1 .

\section{Pathology}

At necropsy the hearts were hypertrophied with prominent left ventricular hypertrophy except for case 5 , in which heart weight was not recorded (table). The endocardium was slightly thickened and opaque @ with pale pink pallor of the myocardium (fig 1). There $\stackrel{3}{\mathcal{S}}$ were no cardiac malformations. The other organs showed changes secondary to congestive cardiac fail- $\infty$ ure.

Light microscopic examination of the myocardium showed some fatty vacuolation throughout the depth

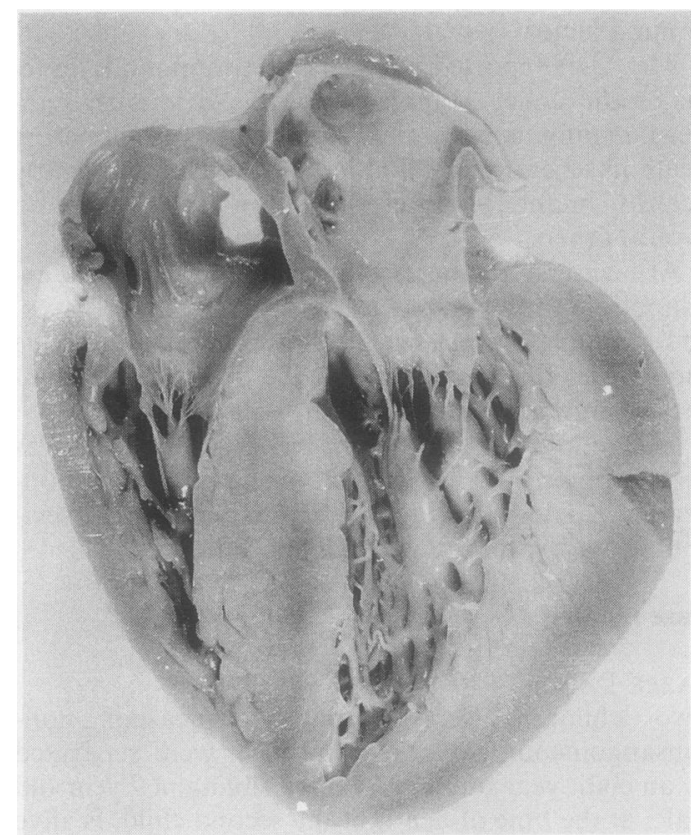

Fig 1 Case 2: longitudinal slice through heart showing ventricular hypertrophy and pallor, especially in subendocardial zone. 
of the wall in most areas. These stained positively with oil red 0 . The cytoplasm had a granular appearance and did not stain for glycogen, except for the normal small amount in the subendocardial zone. The appearances were similar in all the chambers but tended to be most obvious in the left ventricle. The granular cytoplasm gave a positive reaction with the two mitochondrial stains (fig 2).

Ultrastructurally the myocytes contained large numbers of mitochondria in wide pools between scanty, morphologically normal, myofibrils (figs 3 and 4). The mitochondria were concentrated centrally in the myocytes and not under the plasma membrane. As well as being unusually numerous, the mitochondria were rounder and larger than normal. Their maximum diameter was about $1.9 \mu \mathrm{m}$, but many were more than $1.25 \mu \mathrm{m}$. Many contained small dense bodies which correspond to the mainly calcified bodies observed in several conditions, ${ }^{5}$ including various caridomyopathies. A few mitochondria contained parallel arrays of microtubules $700 \mathrm{~nm}$ in length. Most of the mitochondria showed rounded dilatations of the cristae. Several mitochondria contained rounded aggregates of glycogen.

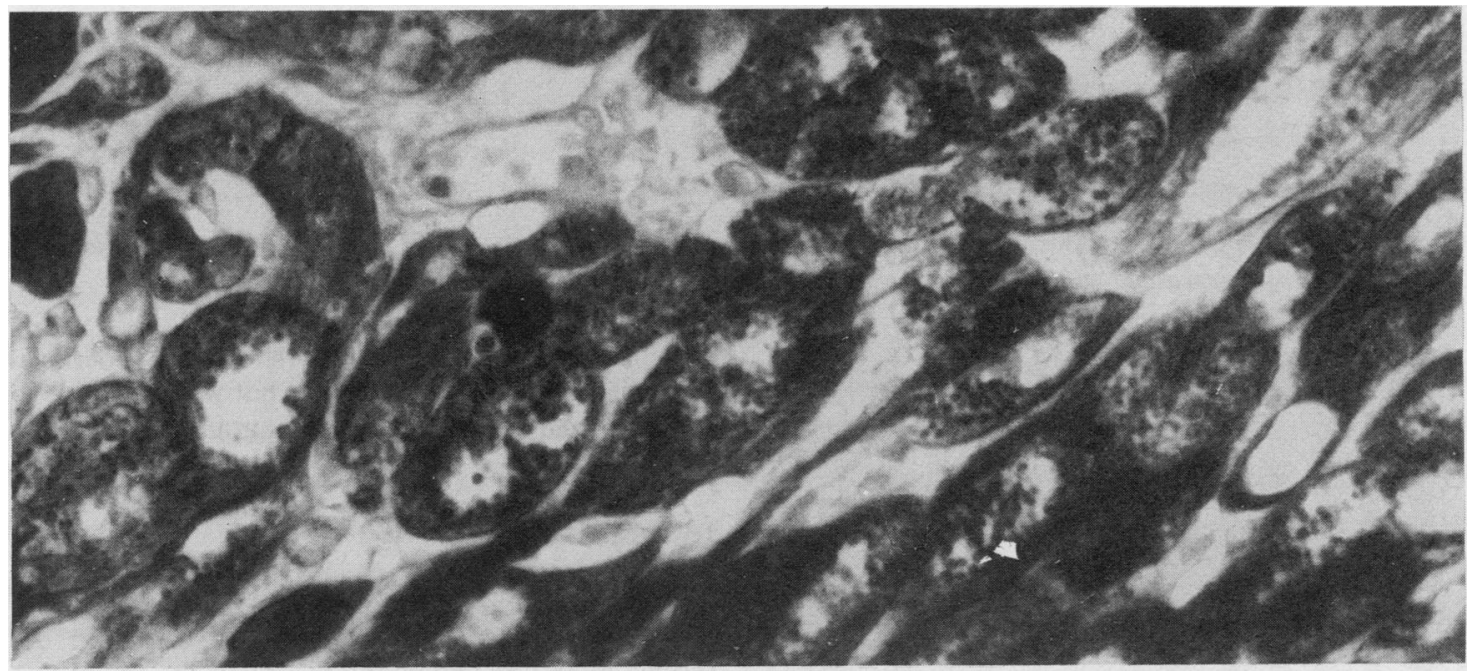

Fig 2 Case 2: myocardial mitochondria. (Modified Swank-Davenport.) $\times 650$.

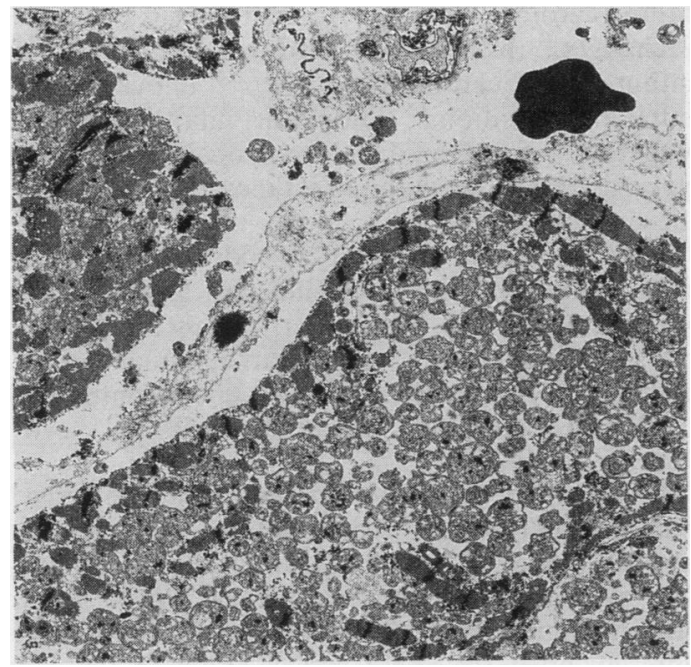

Fig 3 Case 2: myocytes filled with mitochondria and peripherally displaced myofibrils. $\times 2600$.

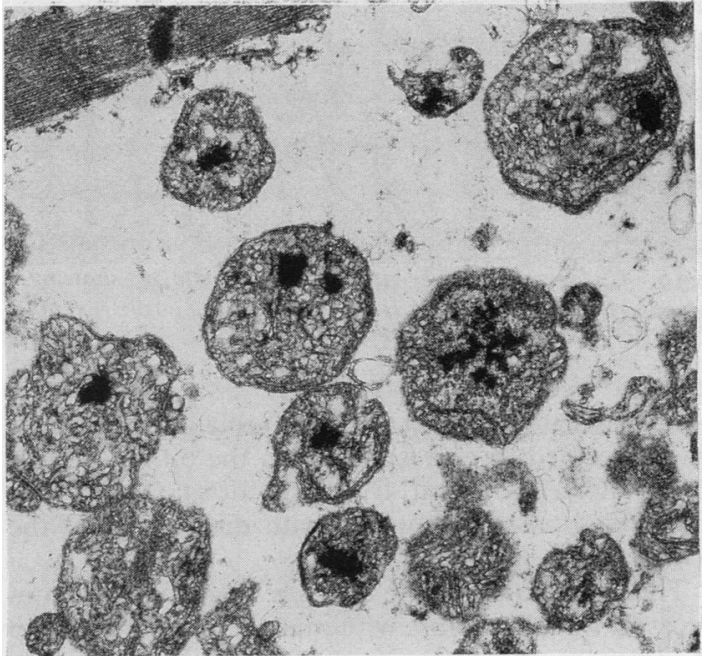

Fig 4 Case 2: myocyte with round large mitochondria with dense inclusions. $\times 16500$. 


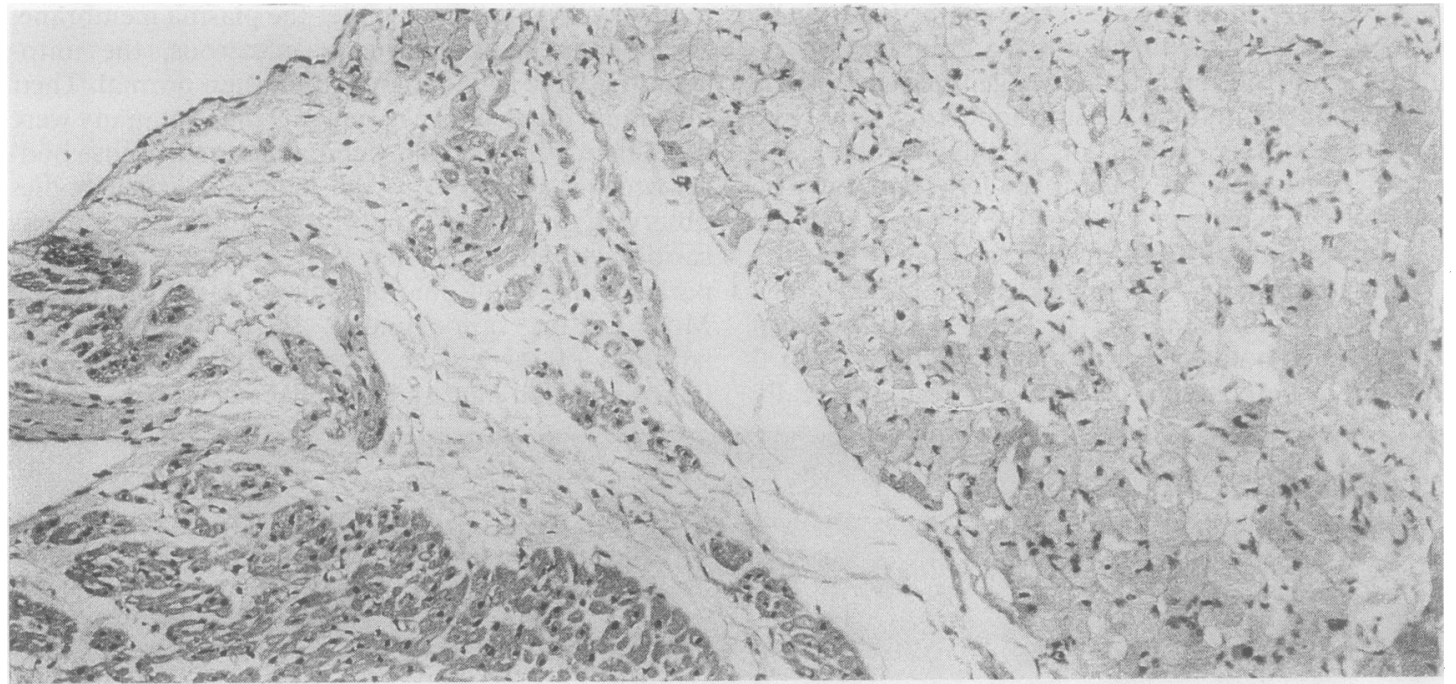

Fig 5 Case 5: subendocardial ventricular myocytes with foamy cytoplasm on right and normal myocytes on left.

(Haematoxylin and eosin.) $\times 125$.

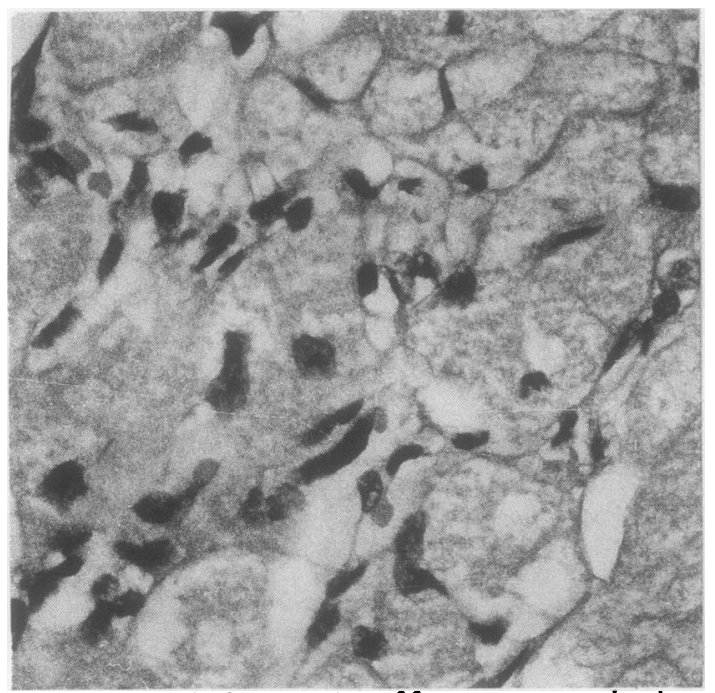

Fig 6 Case 5: high power view of foamy myocytes showing granularity of cytoplasm. (Haematoxylin and eosin.) $\times 500$.

Lipid vacuoles, often with a double limiting membrane, seemed to correspond with the oil red 0 positive material seen on frozen sections. They were interpreted as mitochondria with dissolution of the cristae.

The distribution of the changes in the myocardium in case 5 was less diffuse with numerous focal areas up to $3 \mathrm{~mm}$ in diameter in the trabecular and subendocardial zones of the left ventricle where the myocytes were large with abundant granular eosinophilic cytoplasm (figs 5 and 6). They showed a superficial resemblance to rhabdomyomata but were negative to periodic acid Schiff, were more granular, and less vacuolated.

\section{Discussion}

Foamy myocardial transformation of infancy is a rare condition of unknown aetiology. It has been described mainly in female infants under the age of two years and as isolated cases. It has been referred to by various names, including isolated cardiac lipidosis, ${ }^{6}$ infantile cardiomyopathy with histiocytoid reaction ${ }^{7}$ or changes, ${ }^{8}$ histiocytoid cardiomyopathy, ${ }^{391011}$ xanthomatous cardiomyopathy, ${ }^{12-14}$ focal myocardial degeneration, ${ }^{15}$ idiopathic infantile cardiomyopathy, ${ }^{16}$ focal lipid cardiomyopathy, ${ }^{17}$ foamy myocardial transformation of infancy, ${ }^{18} 19$ and oncocytic cardiomyopathy. ${ }^{20}$

These cases present either as sudden deaths, or, more commonly, with acute illnesses lasting a few hours or days. In the latter instances electrocardiography shows a variety of arrhythmias including runs of supraventricular and ventricular tachycardia and Wolff-Parkinson-White syndrome. ${ }^{136-9121315-1820}$

The principal necropsy findings are in the heart, which is usually grossly hypertrophied. Yellow nodules or a more diffuse yellow discolouration are found in the myocardium, often as subendocardial lesions. These are mainly found in the left ventricule but also commonly affect the right ventricle and both atria. In our four familial cases the left ventricle was diffusely hypertrophied and had a pinkish pallor rather than a 
yellowish discolouration. In case 5 the changes were focal and somewhat nodular but were not evident on macroscopic examination.

Conventional light microscopic examination shows large myocardial fibres with granular cytoplasm. Stains for mitochondria are positive, and there may also be a few lipid globules. It has been suggested that the lipid may be derived from mitochondrial membranes. ${ }^{16}$ This view is supported by our ultrastructural findings of membrane bound fat vesicles in the mitochondria. Electron microscopic examination shows swollen cells distended by large numbers of centrally located mitochondria. At the periphery of the cells there are myofibrils, leptofibrils, and occasional lipid vacuoles. Most workers regard these cells as changed myocardial muscle cells. Our cases showed a similar appearance to those previously described.

Twenty one of the 26 reported cases were female (where sex was stated), and no other affected members of the family were described. This female predominance has led some authors ${ }^{3}$ to suggest a possible X-linked disorder.

Other authors ${ }^{89}$ have implicated a viral mechanism as an aetiological factor. In some instances there was a prodromal illness, which, they suggested, may have been due to a viral infection; this resembles the presentation of case 5 . These proposed aetiological factors do not explain the female predominance or the cardiac hypertrophy.

Silver et al $^{20}$ described a case with oncocytic change in several organs, including salivary gland, and have postulated an early antenatal rubella infection as the cause, with subsequent arrest in development of myocytes in the myocardium. Our familial cases are unlikely to be due to this, although we cannot rule out a non-specific effect of different viruses in early pregnancy.

Our familial cases occurred in two sets of siblings, one set male and the other female. Both showed the morphological features of foamy myocardial tranformation of infancy on gross, light microscopic, and electron microscopic examination. The mother in family B had four normal children with two different sexual partners prior to the current marriage, which had resulted in three liveborn children, two of whom died of foamy myocardial transformation of infancy and one of whom died of encephalitis with no evidence of cardiac disease on gross, light microscopic, or electron microscopic examination. Family A had three liveborn children; the surviving sibling was seven years old at the time of writing.

From the family histories of these four cases it would seem likely that foamy myocardial transformation of infancy may be an inherited condition, possibly due to an autosomal recessive gene rather than an X-linked condition, as has been previously suggested. A simple autosomal recessive condition, however, cannot explain the female predominance of the reported cases.

It is possible that the changes of foamy myocardial transformation represent the end result of injury to the myocardium. Different aetiological factors may be responsible for similar appearances in the heart of susceptible subjects. This might account for some of the apparent disparities in the presentation of the reported cases and the differences in distribution of the foamy change within the myocardium.

The Swank-Davenport method for degenerate myelin fibres ${ }^{21}$ was successfully adapted to show mitochondria in case 2 . The lipid in the membranes of the mitochondria is responsible for the positivity, and, although non-specific, has also proved useful in oncocytomas.

We thank Mrs C Price for typing the manuscript.

References

1 Voth D. Uber die Arachnocytose des Herzmuskels. Frankfurter Zeitschr Path 1962;71:646-8.

2 Hudson REB. Cardiovascular pathology. Baltimore: Williams and Wilkins Co, 1969:1289.

3 Bruton D, Herdson PB, Becroft DMO. Histiocytoid cardiomyopathy of infancy: an unexplained myofibre degeneration. Pathology 1977;9:115-22.

4 Copolleta JM, Wolbach SB. Body length and organ weights of infants and children. Am J Pathol 1933;9:55-70.

5 Ghadially FN. Ultrastructural pathology of the cell and matrix. London: Butterworths, 1982:136-8.

6 Ross CF, Belton EM. A case of isolated cardiac lipidosis. $\mathrm{Br}$ Heart J 1968;30:726-8.

7 Reid JD, Hajdu SI, Attah E. Infantile cardiomyopathy: a previously unrecognised type with histiocytoid reaction. J Pediatr 1968;73:335-9.

8 Ferrans VJ, McAllister HA, Haese WH. Infantile cardiomyopathy with histiocytoid change in cardiac muscle cells. Circulation 1976;53:708-19.

9 Amini M, Bosman C, Marino B. Histiocytoid cardiomyopathy in infancy: a new hypothesis? Chest 1980;77:556-8.

10 Zimmermann A, Diem P, Cottier H. Congenital "histiocytoid" cardiomyopathy: evidence suggesting a developmental disorder of the Purkinje cell system of the heart. Virchows Arch (Pathol Anat) 1982;3\%:187-95.

11 Saffitz JE, Ferrans VJ, Rodriguez ER, Lewis FR, Roberts WC. Histiocytoid cardiomyopathy: a cause of sudden death in apparently healthy infants. Am J Cardiol 1983;52:215-7.

12 MacMahon HE. Infantile xanthomatous cardiomyopathy. Pediatrics 1971;48:312-5.

13 Radford DJ, Chalk SM. Infantile xanthomatous cardiomyopathy. Aust Paediatr J 1980;16:123-5.

14 Cunningham NE, Stewart J. A rare cause of cot death-infantile xanthomatous cardiomyopathy. Med Sci Law 1985;25: 149-52.

15 Haese WH, Maron BJ, Mirowski M, Row RD, Hutchins GM. Peculiar focal myocardial degeneration and fatal ventricular arrhythmias in a child. New Engl J Med 1972;287:180-1.

16 Kauffman SL, Chandra N, Paress NS, Rodriguez-Torres R. Idiopathic infantile cardiomyopathy with involvement of the conduction system. Am J Cardiol 1972;30:648-52.

17 Bove KE, Schwartz DC. Focal lipid cardiomyopathy in an infant 
with paroxysmal atrial tachycardia. Archives of Pathology 1973;95:26-36.

18 Witzleben CL, Pinto M. Foamy myocardial transformation of infancy. Arch Pathol Lab Med 1978;102:306-11.

19 Becker AE, Anderson RH. Pathology of congenital heart disease. London: Butterworths, 1981:407-12.

20 Silver MM, Burns JE, Sethi RK, Rowe RD. Oncocytic cardiomyopathy in an infant with oncocytosis in exocrine and endocrine glands. Hum Pathol 1980;11:598-605.
21 Culling CFA. Handbook of histopathological and histochemical techniques. 3rd Ed. London: Butterworths, 1974:259-60.

Requests for reprints to: Dr Valerie Suarez, Department of Histopathology, The General Hospital, Birmingham B4 6NH, England. 